\section{LE MASCHERE AUTOBIOGRAFICHE DI GRAZIA DELEDDA}

GRAZIA DELEDDA'S AUTOBIOGRAPHICAL MASKS

Alessandra Sanna

Universidad de Granada

Riassunto:

Abstract:

All'interno della produzione di Grazia Among Grazia Deledda's characters, Cosima Deledda, Cosima è il personaggio più is the most autobiographical one, as we can autobiografico, come si può notare dagli notice from the examples. Concordance can esempi riportati. È anche possibile scorgere be noticed between the author and the female assonanze tra l'autrice ed altre tre figure characters of her works: Regina is the main femminili protagoniste delle sue opere: character of Nostalgie, Nina in Il Paese del Regina, protagonista di Nostalgie, Nina di $v$ Il Paese del Vento e Maria Concezione di La della solitudine.

chiesa della solitudine.

Parole chiave:

\section{KEY WORD:}

Deledda, autobiographical, Cosima Deledda, autobiografico, Cosima, personaggi. characters.
Come accennato nel riassunto che precede questo mio breve intervento, Cosima nella vasta produzione di Grazia Deledda rappresenta la maschera più famosa e forse anche quella più trasparente dietro la quale si cela la scrittrice di Nuoro. È un testo che appartiene alla fase più matura della sua produzione, uscito postumo sulla Nuova Antologia (settembre-ottobre 1936) e pubblicato successivamente da Treves nel 1937 con il titolo di Cosima, quasi Grazia, è una delle migliori opportunità che abbiamo di conoscere la scrittrice per la sua carica visibilmente autobiografica. Ciò che Deledda ci lascia è un prezioso manoscritto che si compone di 276 cartelle, con poche correzioni, ma al quale manca la parola "fine" che invece era solita apporre quando considerava che il lavoro non richiedesse ulteriori revisioni.

Antonio Baldini ha corredato il testo della Treves di note biobibliografiche che hanno la funzione di collegare episodi della storia narrata a fatti accaduti all'autrice: gran parte dei nomi dei personaggi sono fedeli alla realtà (iniziando da Cosima che è il secondo nome di battesimo della scrittrice), così come sono riconoscibili i luoghi nei quali si svolge la narrazione (Nuoro, il monte Orthobene ed altri). L'autrice confeziona una sorta di resoconto autobiografico nel quale ci porta in viaggio per la sua vita ripercorrendo i momenti più significativi (la morte del padre, i primi amori, e l'arrivo dei primi successi letterari) fino alla partenza da Nuoro, episodio che conclude il racconto. Per questa ragione affida la narrazione a Cosima alter ego di Grazia, la quale attraverso lo schermo delle terza persona, ci mostra il suo microcosmo da una prospettiva intima e privilegiata dando vita anche ad autoironiche descrizioni come questa:

(...) E dunque alla nostra Cosima salta nella testa chiusa ma ardita di mandare una novella al giornale di mode, con una letterina piene di graziose esibizioni, come, per esempio, la sommaria dipintura della sua vita del suo ambiente, delle sue perpirazioni, e soprattutto con forti e prodi promesse per il suo avvenire lette sue aspirazioni, e soprattutto con forti e prodi promesse per il suo avvenire letterario. fanciulla pressapoco simile a lei, buon poeta che presiedeva al mondo femminile artificiosetto del giornale di moda, e col cuore di lui le porte della fama. Fama che come una bella medaglia aveva il suo rovescio segnato da una croce dolorosa: poiché se il direttore dell' Ultima Moda nel pubblicare la novella presentò al mondo dell'arte, con nobile slancio, la piccola scrittrice, e subito la invitò a mandare altri lavori, in paese la notizia che il nome di lei era apparso stampato sotto colonne di prose ingenuamente dialettale, e che, per maggior pericolo, parlavano di avventure arrischiate, destò una esecrazione unanime e implacabile (Deledda 2005: 82)

La lettera alla quale si riferisce è:

le farò la mia silhouette in due o tre o righe. Ho vent'anni, sono bruna e un tantino.. anche brutta, non tanto però come sembro nell'orribile ritratto in prima pagina $\mathrm{di}$ "Fior di Sardegna". Sono una modestissima signorina di provincia che ha molta 
volontà e coraggio in arte, ma anche nella sua vita, solitaria e silenziosa, è la più timida ragazza del mondo (Scano 1972: 287).

Pare quasi superfluo chiarire che la sola lettura del romanzo in chiave biografica sarebbe riduttiva e non renderebbe merito alla complessità di quest'opera, costruita invece con grande sapienza su un gioco sottile di rimandied allusioni. Cosima rappresenta dunque il punto d'arrivo, la tappa finale di quel "viaggio esperienziale" che Grazia Deledda aveva intrapreso e che aveva trovato spazio dapprima in diverse novelle come I primi passi, Racconti a Grace, Ferro e fuoco, Sotto il pino, Ballo in costume, Medicina popolare ed altre, e successivamente nella forma più complessa del romanzo. Se in Cosima è impossibile non vedere i continui richiami autobiografici a cui abbiamo fatto cenno, in altri casi le assonanze tra l'autrice le protagoniste dei suoi lavori sono più impercettibili.

Si è deciso di limitarci per ovvie ragioni di tempo a parlare di tre figure di donne che per alcuni aspetti si assomigliano alla loro autrice: Regina, protagonista di Nostalgie, Nina di Il Paese del Vento ed infine Maria Concezione personaggio principale dell'ultimo romanzo deleddiano, La chiesa della Solitudine. Nostalgie (1905) inaugura la serie dei lavori cosiddetti "continentali": è il primo cioè che varca i consueti confini dell'isola. Regina, la protagonista, giovane di famiglia nobile ma decaduta, lascia il villino «adagiato sull'argine del Po» (Deledda 1998: 384) per seguire il marito a Roma. La prima similitudine con colei che le ha dato vita è evidente: Regina come Grazia abbandona la terra natia per la capitale che inizialmente è fonte inesauribile di speranze ma anche di timori:

Roma!Un tripudio infantile l'assaliva al solo pensiero che Roma s'avvicinava; che Roma, la città meravigliosa, lungamente sognata, la capitale del mondo, in nido d'ogni delizia e d'ogni splendore, Roma stava per diventar sua! (. . . Ma che cosa saprà darmi Roma? Noi non siamo richi e la grande città è come. poco e dà poco a coloro che non son ricchi... (Deledda 1994: 384)

Roma è la stessa città che sognava Cosima- Grazia quando

nelle sere d'inverno, accanto al bracere e alla luce di due lampadine ad olio, o nei meriggi di primavera, nell'orticello fiorito di rose e ronzante di mosconi, e poi d'estate nella camera su in alto col paesaggio sonnolento dei monti alla finestra (...) Roma era la sua meta: lo sentiva (...) era una specie di città veramente santa, la Gerusalemme dell'arte, il luogo dove si è più vicini a Dio e alla gloria (Deledda 2005: 81).

Deledda si trasferisce a Roma nel marzo del 1900, in seguito al matrimonio con Palmiro Madesani, segretario all'Intendenza delle Finanze: Antonio, marito di Regina è vice-segretario al Ministero del Tesoro, una coincidenza che non ci sembra essere casuale. Nella capitale Grazia spera di trovare una società più moderna e priva di quei pregiudizi e di poter esprimere liberamente la sua arte, possibilità negatele dalla ristretta cerchia culturale sarda prettamente maschile.

Regina non ha ambizioni così alte, tuttavia anche in lei c'è una voglia di superare certe convenzioni, forse anche contagiata dalla cognata Arduina, direttrice del «giornale femminista L'Avvenire della donna» (Deledda 1994: 390), manifesta all'inizio del romanzo il desiderio di ribaltare quel ruolo che tradizionalmente era stato assegnato alla donna nella società del suo tempo:

Lavorare, lavorare! Sì, anch'ella voleva lavorare, voleva scrivere, poiché non era buona ad altro, voleva guadagnare. E anzitutto voleva vivere. “Uscirò dalla cerchia buona ad alno, vole gu a a che mi stringe, guardero la vita in viso. Voghio smarrirmi nelle grandi vie di Roma, o di quelli che sembrano felici e non lo sono: la vita come è (Deledda 1994: 414).

La protagonista di Nostagie non porterà a termine questo progetto, forse perché troppo debole e volubile, mentre l'autrice, in questo senso molto diversa dalla sua "creatura", grazie alla sua caparbietà descriverà Roma in tutte le sue sfaccettature, compresa quella periferica e meno maestosa. Nella novella Cura, ad esempio, la protagonista va in giro con le scarpe più vecchie, nei luoghi più tristi e plebei, per esempio in certe strade sempre fangose e d'un fango nero attaccaticcio, che s'insinuano tra le altre aristocratiche, come l'intestino fra le viscere più nobili (Deledda 2006: 246). mostrando un «interesse tozziano per gli aspetti più cupi della città» (Deledda 2006: 12). Il titolo del romanzo infine, si riferisce proprio al sentimento che accompagnerà la giovane donna durante tutta la narrazione:

Sognò di trovarsi nel bosco, sulle rive del Po, verso Viadana. Un molino scrosciava sulle acque lucenti" (Deledda 1994: 407).

Nonostante sia stato ribadito poc'anzi che Deledda cercò fortementel'allontanamento dalla Sardegna, è altrettanto vero che non spezzò mai quella sorta di "cordone ombelicale" che la teneva legata alla sua terra e che, come sappiamo, ispirò gran parte della sua produzione. Regina rimpiange l'argine del Po e Grazia chiudendo gli occhi rivede i colori della sua Sardegna:

(...) i meravigliosi tramonti di Roma scoloriscono, nel mio ricordo, davanti a quest'immenso orizzonte d'un rosso fragola inverosimile, ove galleggiano, come lontane isole lucenti, lunghe nuvole d'oro, dalle quali s'erge un perfetto miraggio di boschi solitari, immobili nello splendore del tramonto (Deledda 2007: 244).

Diversi paesaggi ma ricordi simili e sentimenti quasi identici. Anche in questo caso, si riscontra una grande similitudine fra personaggio ed autrice.

Passiamo ora a Il paese del vento. Uscito per intero nel 1931, è il romanzo più breve della scrittrice nuorese e tuttavia uno dei più emblematici per quanto riguarda l'aspetto 
che ci interessa. Eurialo De Michelis lo definì «il romanzo più autobiografico della non autobiografica scrittrice; l'unico in prima persona singolare femminile, come nemmeno Cosima» (Deledda 1994: 867). Anche stavolta protagonista della vicenda è una giovane donna, Nina, sposata da appena quindici giorni ad un uomo «civile: vale a dire socievole, di carattere ottimista, inoltre, e quindi fiducioso del suo prossimo che egli ritiene onesto perché onesto è lui» (Deledda 1994: 870). L'incontro con Gabriele, suo primo e platonico amore (si tratta quasi di un'apparizione perché egli è malato e quasi ridotto a "fantasma"), provocherà il risvegliarsi dell'antico sentimento adolescenziale:

(...) mi sentivo spinta da una fatalità simile a quella che spingeva i personaggi del libro che leggevo; ed era sempre il fondo romantico del mio temperamento, quello che agiva, lo sapevo benissimo; ma tentavo di spiegarlo con ragioni mistiche (Deledda 1994: 902)

Nina è tormentata dal senso di colpa nei confronti del leale sposo, ma al contempo si sente a sua volta vittima, perseguitata dalla figura di Gabriele:

(...) la mia pena non era per lui, certamente: in fondo egli continuava a destarmi un po' di paura e molta ripugnanza: la mia pena era per me, che non riuscivo a liberarmi della sua ombra, e ritrovavo qualche cosa di torbido nel mio istinto e di silenzio e di inganno verso la sola persona che realmente, dopo la mia mamma, $\mathrm{m}$ voleva bene (Deledda 1994: 902)

Come sottolinea ancora De Michelis,

la storia di Nina (sognante fanciulla romantica, tutta "assimilata in fantasia", con una sorta di naturale avversione per la prosaica volgarità del reale), la persecuzione di Gabriele, hanno assai poco di autobiografico, ove si voglia pensare a una trascrizione nel narrato di fatti reali, moltissimo invece ove legittimamente, sulla scia dell'ormai lontano Nostalgie, si intreccino riflessi privati di un'adolescenza isolata, piena soprattutto di libri, "turbinosa di sogni e desideri inappagati", turbata dalla pur desiderata partenza, dalle difficolta impreviste, spoetizzanti della nuova vita (De della nuva vita (Dele maniera inequivocabile Grazia si nasconde dietro a Nina:

(...) il temperamento ce l'avevo: nata in un paese dove la donna era considerata ancora con criteri orientali,e quindi segregata in casa con l'unica missione di lavorare e procreare, avevo tutti i segni della razza: piccola, scura, diffidente e sognante, come una beduina che pur dal limite della sua tenda intravede ai confini del deserto i miraggi d'oro in un mondo fantastico, raccoglievo negli occhi il riflesso di questa vastita ardente, di colori liquidi della mia iride (Deledda 1994: 870).

Ritroviamo una Deledda bambina anche nella descrizione della formazione letteraria di Nina:
(...) Eppure mi abbandonavo a quello che la mia mamma considerava il più grosso peccato: la continua avida lettura di libri non adatti alla mia età e soprattutto alla mia educazione. Naturalmente leggevo di nascosto, giorno e notte. In quella camera dove i topolini rosicchiavano le carte, e le rondini facevano i loro primi esercizi di volo, anche la mia anima si apriva lentamente, da sola, ora per ora, foglio per foglio dilibro, mia anima ultimo, nel suo centro, qualche petalo ancora chiuso (Deledda 1994: 874).

Anche la rappresentazione della famiglia della giovane, ricorda molto da vicino quella di Grazia- Cosima: sembrano essere addirittura figlie della stessa madre: quella di Nina «religiosa e austera, non parlava mai, non dava confidenza ai figli: lavorava sempre e usciva di casa solo per andare in chiesa» (Deledda 1994: 875) e quella di Cosima «taciturna, chiusa in un mondo tutto suo; badava ai figli e alle cose domestiche, ma con una freddezza quasi meccanica, con scrupoli di un dovere dal quale non si aspetta nessun premio» (Deledda 2005: 54).

Un altro personaggio di Il paese del vento che ci riporta alla biografia deleddiana è senza dubbio il marito di Nina che ricorda Palmiro Madesani. Deledda lo conobbe nell'ottobre del 1899 a Cagliari, mentre si trovava ospite presso la direttrice del quindicinale La donna sarda Maria Manca. Trentanovenne di origini mantovane ma residente a Roma, Madesani, come si è accennto in precedenza, si trovava sull'isola per lavoro. I due si conoscono a teatro e pochissimo tempo dopo arriva la proposta di nozze. Queste avvengono 1'11 gennaio del 1900, in Santa Maria della Neve, la cattedrale di Nuoro; per l'occasione «La donna sarda» pubblica un fascicolo speciale con una lunga poesia della scrittrice intitolata Viaggio di nozze in Sardegna. Ne Il paese del vento, si racconta l'incontro decisivo, il "gioco d'amore" e la successiva proposta di matrimonio che riceve Nina:

(...) il mio futuro marito si rivolge a me, lisciandosi l'una con l'altra le mani, e scuote la testa quasi per dire a sé stesso: vediamo se l'indovino (...) Così, quando il mio compagno di gioco, per penitenza mi impose di confessare come desideravo fosse il mio futuro sposo, risposi con accento che pareva leggero e invece risonava dal profondo del cuore: "Come lei". Il giorno dopo egli mi scrisse una lettera d'amore: e nel maggio seguente fu celebrato il nostro matrimonio (Deledda 1994: 889).

Questo passo può considerarsi la trascrizione quasi fedele di come la scrittrice di Nuoro e il suo futuro marito iniziarono la loro parabola coniugale, che ricordiamo, contrariamente a quella della coppia del romanzo, non ebbe mai nessun punto d'ombra. Per questa ragione, forse, la coppia destò l'invidia di molti: Luigi Pirandello in una lettera ad Ugo Ojetti confessava: «(...) Son partito dal marito di Grazia Deledda. Lo conosci? Che capolavoro, Ugo mio! Dico, il marito di Grazia Deledda, intendiamoci...» (Zappulla-Muscarà 1980: 28). Lo scrittore siciliano in Giustino Roncella nato Boggiolo (uscito nel 1911 con il titolo più esplicito di Suo marito), descrive un 
uomo totalmente offuscato dalla fama e dalla forte personalità della moglie: tuttavia il carteggio pubblicato in parte dal Momigliano sul Corriere della Sera è la testimonianza della stima e dell'affetto che i coniugi nutrivano l'uno verso l'altra. Madesani non solo accettò di buon grado di avere a fianco una donna "pubblica", ma divenne presto anche l'amministratore più fidato degli affari letterari della scrittrice.

Veniamo ora a Maria Concezione, l'ultima delle donne create dalla penna di Deledda prima che si spegnesse a Roma il 15 agosto del 1936. La chiesa della solitudine esce in quello stesso anno ed è incentrato sul duplice travaglio della giovane protagonista. Colpita da una grave malattia (la stessa che in quello stesso anno condurrà Deledda alla morte), Maria Concezione decide non solo di accettare la disgrazia con rassegnazione ma anche di trasformarla in impedimento: «Capiva benissimo che il suo male era, in rapporto all'amore, come un legame, un voto, un ostacolo simile a tanti altri» (Deledda 1994: 935). Si condanna alla solitudine, rifiutando l'amore del giovane di cui è innamorata, spingendolo seppur involontariamente nelle braccia della sorellastra.

Maria Concezione più che una maschera è un'arma che Deledda fabbrica per "difendersi" dal male che l'aveva attaccata. Un male nascosto con grande riserbo ed esorcizzato anche con l'impegno assiduo nella scrittura; lo stesso male che anche la protagonista del romanzo vuole in tutti i modi occultare. In una lettera dell'agosto del '34 indirizzata alla sorella Deledda scrive: "Io sto relativamente bene, ma sono debolissima e magra come quando da signorina mi mettevo la salvietta sul petto. Se domandano di me dite che sono convalescente da una pleurite e che verrò a Cervia in settembre" (Deledda 2005:14). A sua volta Maria Concezione fa intendere di essers sottoposta "ad una semplice estrazione di un polipo al naso" (Deledda 1994: 934) Questo tentativo di ridimensionare la gravità della malattia, equivale in entrambi i cas alla ricerca della normalità, ad un inconscio rifiuto della realtà, dove la condizione di "malato" viene vissuta come una sorta di colpa che si deve necessariamente nascondere. Ritornano spesso i riferimenti a questa fuga: anche il giovane prete Serafino, amico della sventurata cerca di stimolarla a vivere e a non mettere in scena una inutile farsa:

(...) Tu parli senza convinzione, fingi con me come vuoi fingere con te stessa: così non ci si può intendere. La vita non deve essere commedia Concezione, almeno tra le persone di fede e di giudizio, come io ti ritengo (Deledda 1994: 963).

e lo stesso concetto viene ribadito poco dopo dal dottore:

No cara amica, tu mentisci a te stessa. Basta guardare i tuoi occhi: sembri una zingara mascherata da monaca. E dunque, lasciando le teorie, veniamo alla realtà (Deledda 1994: 977)
Il racconto è condotto da una voce narrante in terza persona che tuttavia è implicata nella vicenda e che in maniera sottile si identifica con la protagonista «ne comprende il travaglio con un'intesa solidale che rivela lucide implicazioni e indiretti richiami biografici» (Deledda 2008: 13).

Nell'intero romanzo sono disseminate citazioni bibliche e tutta la vicenda è scandita temporalmente dalle festività religiose: a dicembre l'Immacolata, giorno in cui Concezione rientra dall'ospedale, in primavera la Passione e la Resurrezione del Cristo, ed infine a luglio la festa del paese, San Cirillo. La chiesa della solitudine rappresenta in questo senso l'ultimo prodotto di quella concezione etico- religiosa che Deledda aveva elaborato nel corso degli anni e che aveva caratterizzato anche molti dei suo lavori precedenti. In diverse occasioni le donne deleddiane invocano la Madre di Dio ne chiedono protezione ed aiuto: anche Maria Concezione si rivolge alla Madonna chiedendole pietà e la forza per dimenticare il suo amore:

Maria, Madre di Dio, fa che mi lascino in pace", pregava Concezione inginocchiata ai piedi dell'altare "non domando che di poter vivere finché vive mia madre, e di non farla soffrire: dopo fa di me quello che tu vuoi. Sono pronta a tutto; non $\mathrm{mi}$ spaventa il dolore, ma il peccato mortale. (...) Maria, Madre di Dio, levatemelo dal pensiero, fate che egli se ne vada lontano nelle altre parti della terra,; che io non senta più sue notizie: che egli sia felice, libero dal peccato, e rimanga buono e puro come l'ho conosciuto io (Deledda 1994: 976).

La parziale identificazione di Grazia con Maria Concezione è secondo il mio parere piuttosto evidente, tuttavia una delle tante "prove" risiede in novella intitolata La chiesa nuova della raccolta Sole d'estate, dalla evidentissima carica biografica:

(...) La chiesa è quella, il bosco è quello. È la chiesetta antichissima, in cima a Monte Orthobene, sopra la cascata dei lecci, nell'ora quando il cielo si sprofonda Monte Orthobe, qupra fino a Dio dal mondo salgono le nuvole rosse che hanno assorbito e disperdono le passioni degli uomini. Sono ancora fanciulla: la vita e dentro il mio pugno, come una manciata di gemme; ma io la depongo ai tuoi piedi, Signora del Monte, come nella canzone nuorese la giovane fidanzata morente offre le sue collane alla Vergine Maria. Tutto io ti offrivo, Regina del Monte purché tu mi conservassi la fede (Deledda 2006: 122).

Giovanna Cerina a proposito di Cosima e de La chiesa della solitudine scriveva:

due romanzi richiamano due fasi diverse, distanti nel tempo ma complementari, libere da implicazioni direttamente autobiografiche, grazie anche alla scelta di un narratore in terza persona e di nuove modalita espressive e stilistiche, abilmente diversificate. Due storie diverse, due personaggi fenminili diversi, che interpretano aspetti riguardanti da vicino esperienze di vita della Deledda: quella euforica di un percorso in ascesa, e l'altra che riguarda il lato notturno della vita, la sofferenza, i dolore, il ripiegamento su di sé. Il lettore trova tutti i segnali e i dati plausibili per riconoscere, in filigrana, le voci dell'anima e i silenzi, le gioie e le inquietudini, i 
tempo del successo e la malattia che hanno segnato la caratura umana e letteraria di Grazia. (Deledda 2008: 10).

In una lettera indirizzata ad Andrea Pirodda Deledda affermava con decisione: «è impossibile scrivere un lungo romanzo senza mettervi qualcosa di vivo, di veduto, di udito, di sentito: ma non ho creato alcuno dei personaggi con lo scopo preciso di ritrarre persone vive» (Di Pilla 1966:382). Tuttavia mi pare di aver dimostrato, concordando con l'opinione di altri critici, che in diversi casi le donne deleddiane si possano interpretare senza alcuna forzatura come il frutto della rielaborazione di esperienze e sentimenti della scrittrice di Nuoro, o più semplicemente, come le maschere autobiografiche di Grazia Deledda.

\section{RIFERIMENTI BIBLIOGRAFICI}

Deledda, G., Dieci romanzi, (a cura di Anna Dolfi), Roma, Newton, 1994.

----, Cosima, (prefazione di Giovanna Cerina), Nuoro, Ilisso, 2005.

----, La chiesa della solitudine, (prefazione di Giovanna Cerina), Nuoro, Ilisso, 2008.

---- "Nostalgie", in Dieci romanzi, op. cit. pp. 381-506.

---- "Il paese del vento", in Dieci romanzi, op. cit. pp. 867-925.

---- "La chiesa della solitudine”, in Dieci romanzi, op. cit. pp. 929-1013.

---- ,Tipi e paesaggi sardi", in AA. VV. La grande enciclopedia della Sardegna.Tradizioni e personaggi leggendari, Sassari, La Nuova Sardegna, pp. 224-252, 2007.

----, "Cura" in Novelle, vol. IV, a cura di Giovanna Cerina, Nuoro, Ilisso, pp. 246-249, 2006.

----, "La chiesa nuova" in Novelle, vol. VI, a cura di Giovanna Cerina, Nuoro, Ilisso, pp. 118-122, 2006.

Di Pilla, F., "La vita e l'opera di Grazia Deledda", in Grazia Deledda Premio Nobel per la letteratura 1926, Milano, Fabbri, 1966.

Scano, A., Versi e prose giovanili, Milano, Virgilio, 1972.

Zappulla Muscarà, S. G., Deledda. Carteggi inediti, Roma, 1980. 